\title{
Navigation Cost Modeling Based On Ontology
}

\author{
${ }^{1}$ Madala Venkatesh, ${ }^{2}$ Dr.R.V.Krishnaiah \\ ${ }^{I}$ Department of Computer Science \& Engineering, DRK institute of science and technology, Hyderabad, India. \\ ${ }^{2}$ Principal, Department of Computer Science \& Engineering, D.R.K Institute of science and technology, \\ Hyderabad, India.
}

\begin{abstract}
Web databases when queried result in huge number of records when users of query need a portion of those results which are real interest to them. This problem can be solved using concept hierarchies. Knowledge representation in the form of concepts and the relationships among them (Ontology) allows effective navigation. This paper presents provisions for categorization and ranking in order to reduce the number of results of query and also ensure that the navigation is effective. User should not spend much time to view the actual subset of records he is interested in from the avalanche of records that have been retrieved. For experiments, PubMed database which is in the public domain is used. The PubMed data is medical in nature and organized as per the annotations provided that is instrumental in making concept hierarchies to represent the whole dataset of PubMed. The proposed technique in this paper provides a new search interface that facilitates end users to have effective navigation of query results that are presented in the form of concept hierarchies. Moreover the query results are presented in such a way that the navigation cost is minimized and thus giving rich user experience in this area. The empirical results revealed that the proposed navigation system is effective and can be adapted to real world systems where huge number of tuples is to be presented.
\end{abstract}

Index Terms - Concept hierarchy, effective navigation, annotated data

\section{Introduction}

The amount of data provided over World Wide Web (WWW) is increasing rapidly every year. In the past decade in started growing drastically. Especially biomedical data and the literature pertaining to it that reviews the aspects of biomedical data across the globe have seen tremendous growth in terms of quantity. Biological data sources such as [2], [3], and [4] are growing in terms of lakhs of new citations every year. The queries made by people associated with healthcare domain have to search such databases by providing a search keyword. The results are very huge in number and the users are not able to view all the records when they actually need a subset of them. This has led to users to refine query with other keywords and get the desired results after many trials. Here it has to be observed that user time is wasted in refining search criteria and also the navigation of query results which are abundant and bulky. The navigation cost is more as user has to spend lot of time in finding the required subset of rows from the bulk of search results. This problem has been researched in [2], [3], [4] and the problem is identified as information overload. Figure 1 shows static navigation of MeSh hierarchy of biomedical data.

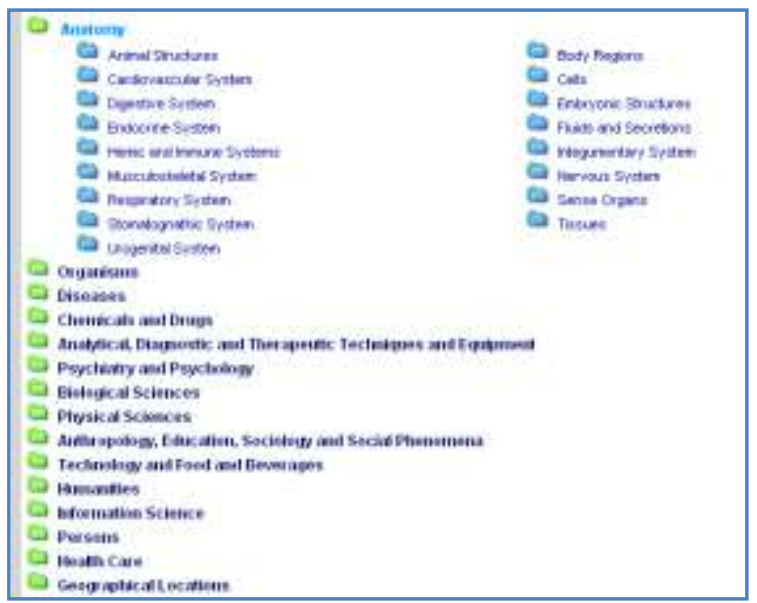

Fig. 1: Static structure of MeSh hierarchy of biomedical data [5]

The solutions are of two types namely categorization and ranking. However, these two can be combined to have more desired results. The proposed system is specially meant for presenting results in such a way that the navigation cost is reduced. For this purpose categorization techniques is used and concept 
hierarchies are built. The categorization techniques are supported by simple ranking techniques. The proposed solution uses citations as described in [8] and effectively constructs a navigation tree that can reduce cost of navigation and user's experience is much better when compared with existing systems that do not use these techniques. These techniques are being used by e-Commerce systems to let their users have smooth navigation to the results returned by such systems.

The proposed system uses a cost model that lets it estimate the cost of navigation and make decisions in providing concept hierarchies. The cost of navigation is directly proportionate to the navigation subtree instead of the whole results in the tree. Earlier work on dynamic categorization of query results are in [3], [4], [10] and [6]. They made use of query dependent clusters based on the unsupervised technique. However, they neglect the process of navigation of clusters. In this aspect the proposed system is distinct and provides dynamic navigation on a pre-defined concept hierarchy. Another telling difference between existing systems and the proposed one is that the proposed system uses navigation cost model that minimizes navigation cost no matter what the bulky of search results is. Overall, our contributions are development of a framework for effective navigation of query results; a formal model for cost estimation; algorithm to optimize the results' navigation cost; experimental evidence on the effectiveness.

\section{Overview Of The Proposed Framework}

MeSH (Medical Subject Headings) is the hierarchy based on which the framework is built. It is a made up of a concept hierarchy. A concept hierarchy is a labeled tree which has concept nodes and edges with a root node. Each node is identified by a unique ID and associated with a label. As discussed in [9], as per the MeSH concept hierarchy the label of parent is not specific when compared with that of child. Almost all concept hierarchies follow the same conventions. We have built a prototype application whose interface after search operation is as shown in fig. 2.

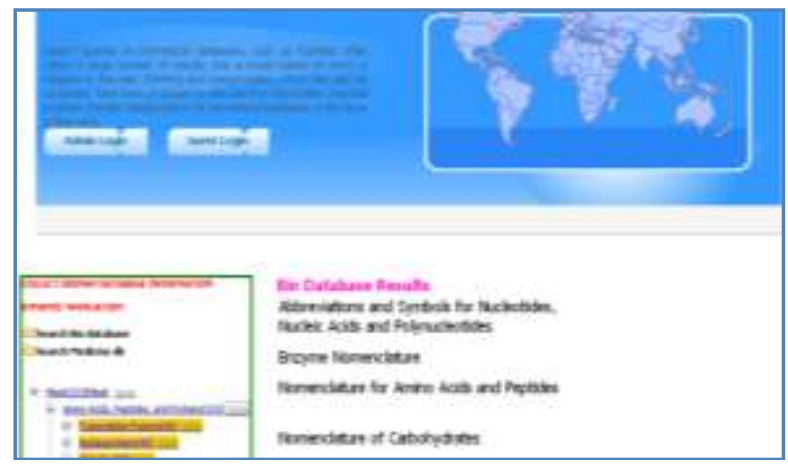

Fig. 2 - Prototype application

When a query made by user, the application returns results in terms of citations list associated with $\mathrm{MeSH}$ concepts. The application constructs an initial navigation tree which is nothing but a concept hierarchy. Every node in that hierarchy is known as a concept. The navigational tree presented initially my contain nodes that are empty. As MeSH is very large hierarchy, the proposed application removes empty nodes and thus reduces size of the navigation tree. The removal of empty node is done carefully by preserving node relationships. The resultant navigation tree is nothing but the initial navigation tree where the empty nodes have been removed to reduce the size of the tree.

By removing empty nodes from the navigation tree, the tree size is reduced. However, the structure of the navigation tree is very big and can't be presented to end user as it is. The proposed application automatically expands the tree at required place to ensure that user navigation cost is less and user will directly see the desired results. Towards this end the application takes care of hiding unnecessary nodes and presenting desired nodes in such a way that the navigation cost of to the user is negligible. We consider a node expansion at given place as an "EdgeCut". In case of trees, in general an EdgeCut is a subset of edges because the removal of edge causes a new component to be created. Visualization of an EdgeCut is shown in fig. 3.

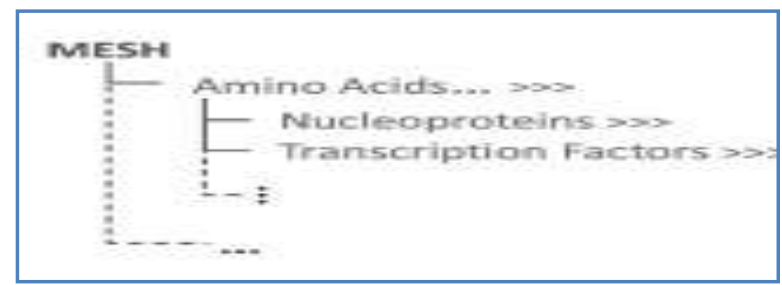

Fig. 3 - Visualization of EdgeCut [1] 
Navigation tree with an EdgeCut is shown in fig. 9 on the user interface. The EdgeCut results in creation of sub trees and they are of two types namely lower and upper. Before an EdgeCut is performed, the navigation tree is converted into something known as active tree. It is achieved by annotating root node. The navigation tree is reduced both width and height wise due to the EdgeCut and presentation of the resultant tree. We do assume any user's preference on the results and every part of the tree can be reached by user as the navigation does not result in information loss in the proposed framework. We also use a convention i.e., " >>>" used to provide hyperlinks that facilitate user to perform EdgeCut operations in recursive manner. Generally we expect EdgeCut to be triggered by user on the lower component of sub trees. However, it is also possible that such operation may occur at upper component sub tree. Fig. 4 shows the resultant tree with hyperlinks in ">>>" convention.

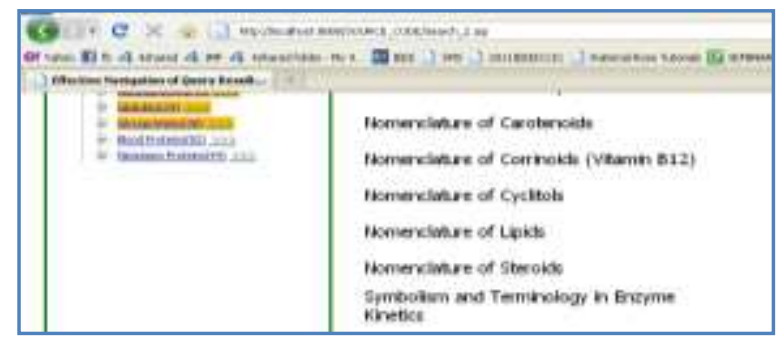

Fig. 4 - Resultant Tree

\section{Architecture Of Prototype System}

The architecture of the prototype application is as shown in fig. 5. The architectural operations are classified into online and offline operations.

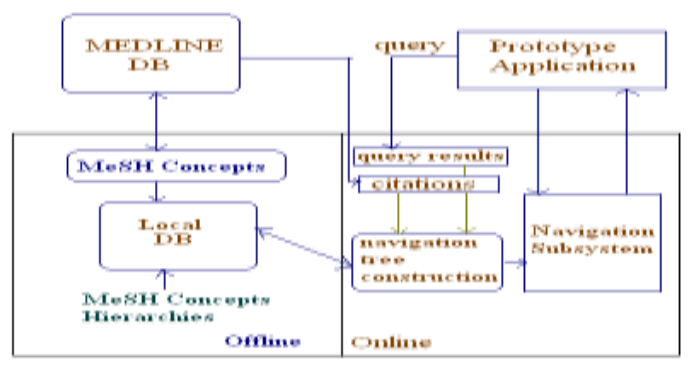

Fig. 5 - Architecture of Proposed Application

As seen in fig. 5, the proposed system operations are classified into online and offline operations. The offline operations include storing MeSH concepts hierarchies into local database, exchanging MeSH concepts between PubMed DB and Local DB. The online operations include question results generation, obtaining citations from MEDLINE database. Navigation tree construction and other navigation sub system operations such as active tree visualization are also online operations. The online operations actively start with query given by end user on biomedical database.

\section{Navigation And Cost Model}

Once user issues a query keyword, the prototype application generates an initial active tree with single component tree. Its root is the root of the MeSH hierarchy. Later on user performs one of the following operations.

EXPAND: It takes place when user clicks " $>>>$ " hyperlink that causes EdgeCut to be performed that shows a set of nodes.

SHOWRESULTS: User performs this operation to view results.

IGNORE: User can simply ignore a node after looking at its label and moves to next concept.

BACKTRACK: This action occurs when user performs undo on the last EdgeCut operation.

User continues these operations until he gets the intended results. We simplify this simple navigational model and call it "TOPDOWN". The TOPDOWN model has only three operations namely EXPAND, SHOWRESULTS and IGNORE.

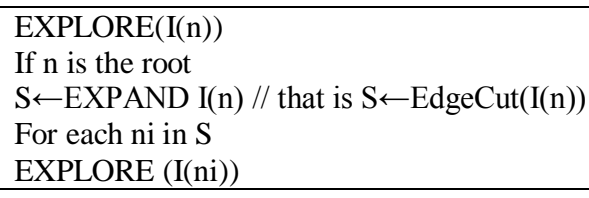


else, if $\mathrm{n}$ is not a leaf -node, choose one of the following:

1. SHOWRESULTS I(n)

2. IGNORE I(n)

3. $\quad \mathrm{S} \leftarrow$ EXPAND I(n)

For each ni in $\mathrm{S}$

EXPLORE(I(Ni))

Else, choose one of the following: // $\mathrm{n}$ is a leaf node

1. SHOWRESULTS I(n)

2. IGNORE I(n)

Listing 1 - TOPDOWN Navigation Model

The cost model as specified in [2] considers the following to compute navigation cost.

- Number of EXPAND actions.

- Number of concept nodes shown by a single EXPAN action.

- Number of citations presented for a single SHOWRESULTS action.

For each of these things cost of 1 is considered. The cost of exploring a component sub tree I(n) rooted at node $n$ is $\operatorname{cost}(\mathrm{I}(\mathrm{n}))=$

$\mathrm{P}^{\mathrm{N}}(\mathrm{I}(\mathrm{n})) \cdot\left(1-\mathrm{P}_{\mathrm{c}}(\mathrm{I}(\mathrm{n})) \cdot|\mathrm{L}(\mathrm{I}(\mathrm{n}))|\right.$

$$
\left.+\mathrm{P}_{\mathrm{c}}(\mathrm{I}(\mathrm{n})) \cdot\left(\mathrm{B}+|\mathrm{S}|+\sum_{\mathrm{s} \in \mathrm{S}} \operatorname{cost}\left(\mathrm{I}_{\mathrm{C}}(\mathrm{S})\right)\right)\right)(1)
$$

where normalized $P_{E}(I(n))$ is represented as $P_{E}^{N}(I(n))$ thus the sum of normalization of sub tree of the component after an EdgeCut is equal to 1.

\section{Best Edgecut Algorithms}

Optimal cost can be computed by recursively listing all possible sets of EdgeCuts. This starts from the root and traverses every concept in the tree. This algorithm is expensive. To overcome this Opt EdgeCut algorithm is proposed which provides minimum expected navigation cost.

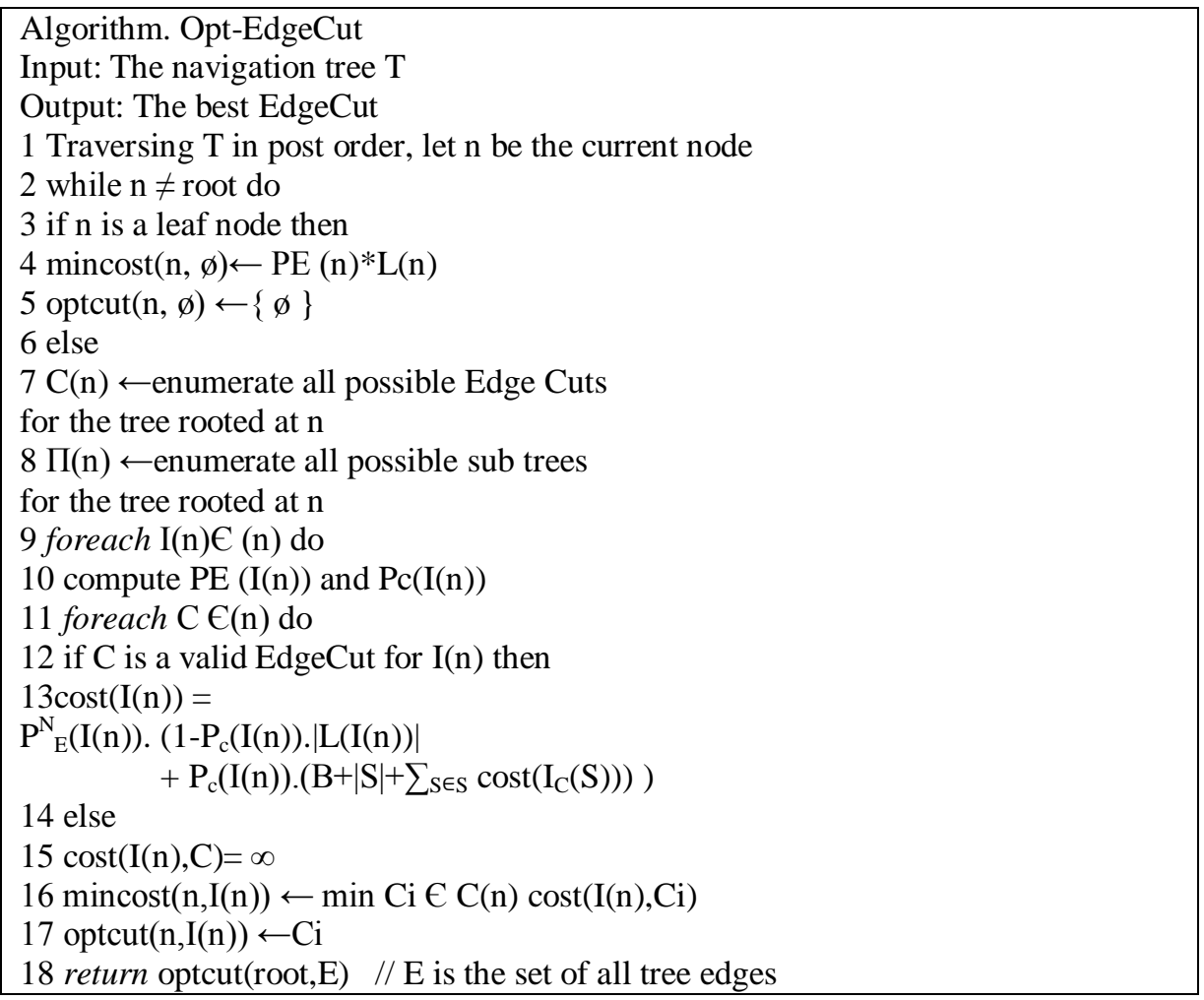

Listing 2 - Opt-EdgeCut Algorithm

The algorithm Opt-EdgeCut is supposed to compute the minimum expected navigation cost required for navigating the tree from bottom up in post order fashion. 


\section{Heuristic-ReducedOpt Algorithm}

The Opt-EdgeCut algorithm is computational more expensive and can't be practically used for most of the queries. Therefore, we proposed a new algorithm known as Heuristic-ReducedOpt as shown in listing 3.

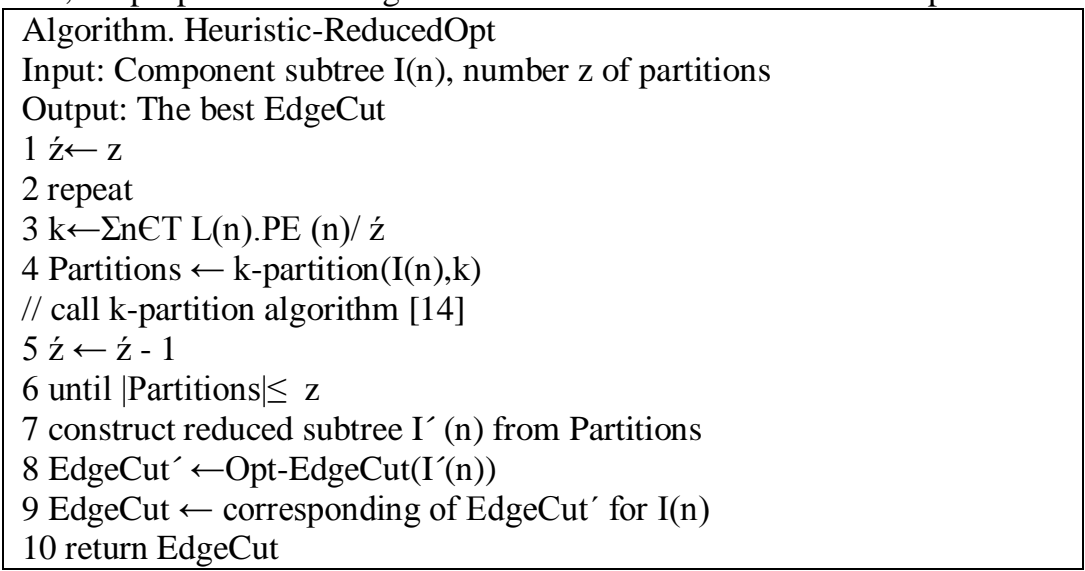

$$
\text { Listing } 3 \text { - Heuristic ReducedOpt Algorithm }
$$

This algorithm is based on k-partition algorithm [10]. This is adapted for our use here. The algorithm works in bottom-up fashion. For every node (n), the algorithm prunes heaviest children one by one till the weight of $\boldsymbol{n}$ falls below k.

\section{Experimental Evaluation}

For evaluating the proposed application, expansion time performance and average navigation cost are considered. The empirical studies are made in a PC with XP as operating system. Oracle $10 \mathrm{~g}$ is used as backend and Java is used to implement all algorithms. The proposed application achieves improvement in navigation cost when compared with Top1-Leave|Wise. Minimum improvement is 16 percent and maximum improvement is 41 percent. Fig. 6 shows number of EXPAND actions comparison.

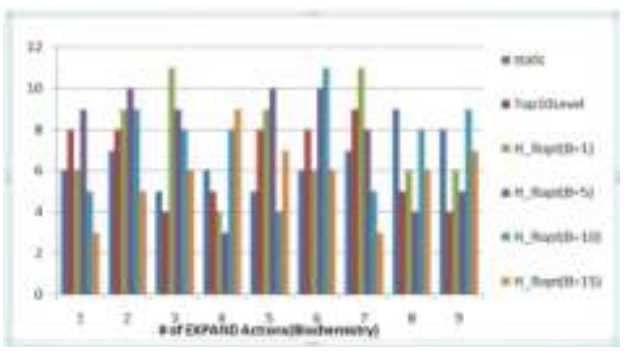

Fig. 6 - Comparison of number of expand operations

As can be seen in fig. 6, ten queries have been presented for six types of algorithms. The $\mathrm{X}$ axis takes question numbers while the $\mathrm{Y}$ axis represents value. Out of all the algorithms the proposed application and its algorithm. Fig. 7 shows the results of number of concepts shown.

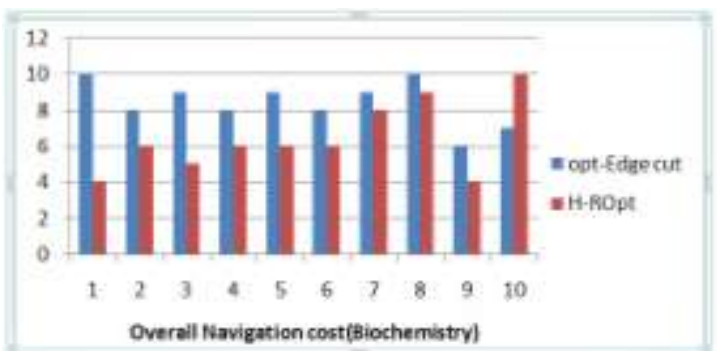

Fig. 7 - Comparison of number of concepts revealed

Fig. 7 shows the number of concepts shown when an EXAPND action takes place. The results revealed that our approach is superior to many other approaches. 


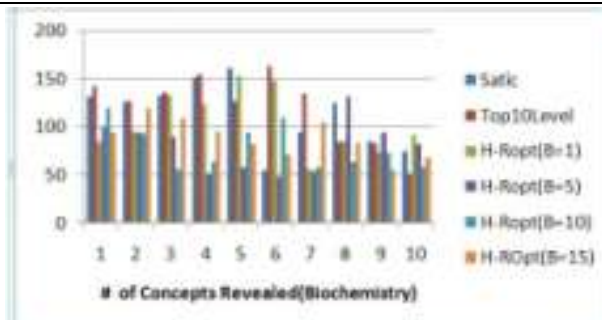

Fig. 8 - Comparison of overall navigation cost

As can be seen in fig. 8 comparison is made on proportional navigation cost of Heuristic-ReducedOpt over Opt-EdgeCut. Opt-EdgeCut algorithm has best results when executed on biochemistry database.

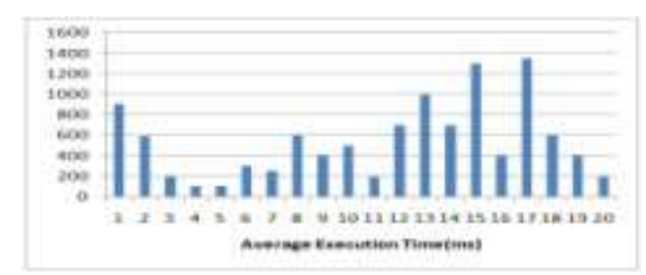

Fig. 9 - Heuristic-ReducedOpt EXPAND performance.

As seen in fig. 9, the average time of Heuristic-ReducedOpt to execute and EXPAND action with respect to each query of table 1. The average values are taken from the number of EXPAND action provided in fig. 6 .

\section{Conclusion}

This paper presents a framework for effective navigation of results of query given to biomedical databases such as PubMed. The problem with query results is that biomedical database returns millions of records and users have to spend some time to navigate to the desired records in the results. This is known as navigation cost. Such problem is also known as information overload problem. The aim of the proposed framework is to address the problem by reducing navigation cost. We achieve this by organizing the results based on the associated MeSH (Medical Subject Headings) hierarchy by proposing a method that works on the resulting navigation tree. The method is known as dynamic navigation method. After applying this method, every node when expanded reveals a subset of required rows thus reducing navigation cost. We have described the underlying cost models and also evaluated them. We developed a prototype application to test the framework's functionality. The empirical results revealed that the proposed framework is effective and can be used in the real time applications.

\section{References}

[1] Abhijith Kashyap, Vagelis Hristidis, Michalis Petropoulos, and Sotiria Tavoulari (2011), "Effective Navigation of Query Results Based on Concept Hierarchies". IEEE TRANSACTIONS ON KNOWLEDGE AND DAT A ENGINEERING, VOL. 23, NO. 4.

[2] J S. Agrawal, S. Chaudhuri, G. Das and A. Gionis: Automated Ranking of Database Query Results. In Proceedings of First BiennialConference on Innovative Data Systems Research (CIDR),2003.

[3] K. Chakrabarti, S. Chaudhuri and S.W. Hwang: Automatic Categorization of Query Results. SIGMOD Conference 2004: 755-766.

[4] Z. Chen and T. Li: Addressing Diverse User Preferences in SQLQuery- Result Navigation. SIGMOD Conference 2007: 641-652.

[5] HON (2010): Health On the Net Foundation: Medical information. Available online at: http://www.hon.ch/cgibin/HONselect?cat+A [viewed: 15 August 2012]

[6] A. Kashyap, V. Hristidis, M. Petropoulos, and S. Tavoulari: BioNav: Effective Navigation on Query Results of Biomedical Databases. (Short Paper), ICDE 2009, to appear. Available at http://www.cs.fiu.edu/ vagelis/publications/BioNavICDE09.pdf S. Kundu and J. Misra, “A Linear Tree Partitioning Algorithm,” SIAM J. Computing, vol. 6, no. 1, pp. 151-154, 1977. Medical Subject Headings (MeSH®). http://www.nlm.nih.gov/mesh/

[9] Medical Subject Headings (MeSH), http: //www.nlm.nih.gov/ mesh/, 2010.
[10] Vivísimo, Inc. -Clusty. [Online].Available: http://clusty.com/,2008

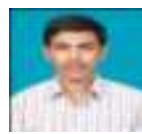

Madala Venkatesh has received B.Tech degree in Computer Science and Engineering and persuing M.Tech in Computer Science and Engineering. His main research includes Data Mining and Cloud Computing.

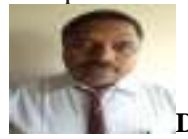

Dr. R. V. Krishnaiah has received Ph.D from JNTU Ananthapur, M.Tech in CSE from JNTU Hyderabad, M.Tech in EIE from NIT (former REC) Warangal and B.Tech in ECE from Bapatla Engineering College. His main research interest includes Data Mining, Software Engineering.. 